\section{CARDIAC RESYNCHRONISATION THERAPY LEADS TO REVERSAL OF HEART FAILURE INDUCED SARCOPENIA}

${ }^{1}$ David Warriner*, 'Philip Webb, ${ }^{2}$ Paul Sheridan, 'Patricia Lawford. 'Sheffield University; ${ }^{2}$ Chesterfield Royal Infirmary; *Presenting Author

10.1136/heartjnl-2016-309890.2

Background Sarcopenia, a reduction in skeletal muscle performance, is common in heart failure (HF) and grip strength (GS) is a measure of such. No trial has investigated the role of cardiac resynchronisation therapy (CRT) in reversing sarcopenia.

Methods GS was measured at baseline and 12 months following CRT. The patient group were 94\% male, mean age $69 \pm$ 8 years, New York Heart Association (NYHA) functional class II-IV, QRSd $173 \pm 21 \mathrm{~ms}$ and had a left ventricular ejection fraction (LVEF) $26 \pm 8 \%$.

Results $70 \%$ of patients were found to have responded at 12 months. Responders had significant improvements in $\mathrm{VO}_{2}$ $(12.6 \pm 1.7$ to $14.7 \pm 1.5 \mathrm{ml} / \mathrm{kg} / \mathrm{min}, p<0.05)$, quality of life score $(43 \pm 23$ to $24 \pm 22, p<0.01)$, left ventricular end diastolic volume $(210 \pm 125 \mathrm{ml}$ to $173 \pm 125 \mathrm{ml}, p<0.01)$, 6 min walk distance $(379 \pm 117 \mathrm{~m}$ at baseline to $418 \pm 105$ $\mathrm{m}(p<0.05)$ and $\mathrm{N}$-terminal pro-B-type natriuretic peptide $(2422 \pm 829$ to $1732 \pm 976 \mathrm{pg} / \mathrm{ml}, p<0.01)$. GS significantly increased, by over $18 \%$ in responders during follow-up.

Conclusion This study demonstrates responders demonstrate a significant improvement in GS following CRT. This demonstrates that CRT improves not only cardiac performance but also secondary gains in skeletal muscle function.

\section{REPORTING QUALITY OF RANDOMISED CONTROLLED TRIALS INVESTIGATING EFFICACY OF PHARMACOLOGICAL THERAPIES FOR HEART FAILURE WITH PRESERVED EJECTION FRACTION}

${ }^{1}$ Sean Zheng ${ }^{*}$, ${ }^{2}$ Fiona Chan, ${ }^{3}$ Edd Maclean, ${ }^{4}$ Shruti Jayakumar, ${ }^{5}$ Adam Nabeebaccus. ${ }^{1}$ King's College Hospital; ${ }^{2}$ East Sussex Healthcare NHS Trust; ${ }^{3}$ Royal Brompton Hospital; ${ }^{4}$ King's College London Medical School; ${ }^{5}$ King's College London; *Presenting Author

\subsection{6/heartjnl-2016-309890.3}

Introduction Randomised controlled trials (RCT) provide the highest level of evidence on healthcare interventions. Accurate interpretation of results and meta-analysis requires adequate reporting. The CONSORT statement, first published in 1996 and later revised in 2001 and 2010, aims to improve reporting quality of RCTs. Heart failure with preserved ejection fraction (HFPEF) is a major cause of morbidity and mortality, comparable to HF with reduced ejection fraction. While many studies have investigated the efficacy of various pharmacological agents, scarcity of consensus guidelines reflects the conflicting results generated from such RCTs.

The aims of this study are to: 1) systematically identify RCTs investigating the efficacy of pharmacological therapies in HFPEF, 2) assess reporting quality with CONSORT 2010 statement and 3) identify trends in reporting quality.

Methods Medline, EMBASE and CENTRAL were systematically searched from 1 January 1996 to 1 September 2015 using pre-specified inclusion-exclusion criteria (Table 1). Titles and abstracts were assessed for relevance. Full texts were independently assessed by three authors for inclusion. Reporting quality was independently assessed by two authors using the CONSORT 2010 score, with each item scored and weighted
Abstract 3 Table 1 Example search strategy and inclusion/ exclusion criteria

\begin{tabular}{l} 
HFPEF search strategy for Ovid MEDLINE(R) \\
1. Heart failure \\
2. Normal ejection fraction \\
3. Preserved cardiac function \\
4. Preserved ejection fraction \\
5. 1 and 2 \\
6. 1 and 3 \\
7. 1 and 4 \\
8. Diastolic heart failure \\
9. Diastolic dysfunction \\
10. HFPEF \\
11. HFNEF \\
12. or/5-11 \\
Inclusion criteria \\
1. Randomised controlled trial \\
2. Trial inclusion criteria specifying heart failure signs and symptoms, left ventricular \\
ejection fraction $>40 \%$ \\
3. Pharmacological intervention with placebo or other pharmacological comparison \\
4. Outcomes including all-cause and cardiovascular mortality, hospitalisation, changes in \\
NYHA class, exercise capacity (6-min walking distance, V ${ }_{2 \text { Max }}$ ) or quality of life \\
Exclusion criteria \\
1. Non-English language \\
2. Abstracts or conference publication \\
3. Unpublished studies \\
4. Healthy controls \\
\hline
\end{tabular}

equally. An overall reporting quality score was calculated for each study. Statistical analysis was performed with SPSS.

Results Initial search identified 3426 studies; 32 were included in the final analysis. There was inter-observer agreement for 1078 out of 1184 values (91.1\%); Cohen's kappa score for inter-observer variability was 0.85 . The mean score was $55.3 \%$ (range 23.3-93.8\%, SD 17.3\%). Under half of published studies $(41 \%, 13 / 32)$ did not adequately report more than half of all relevant sections. The best reported criteria, where applicable, were protocol referencing (criterion 24) $(100 \%, 12 / 12)$, interim analysis $(7 b)(100 \%, 6 / 6)$, changes to methods $(3 b)(100 \%, 6 / 6)$, statistical methods (12a) $(96.9 \%$,

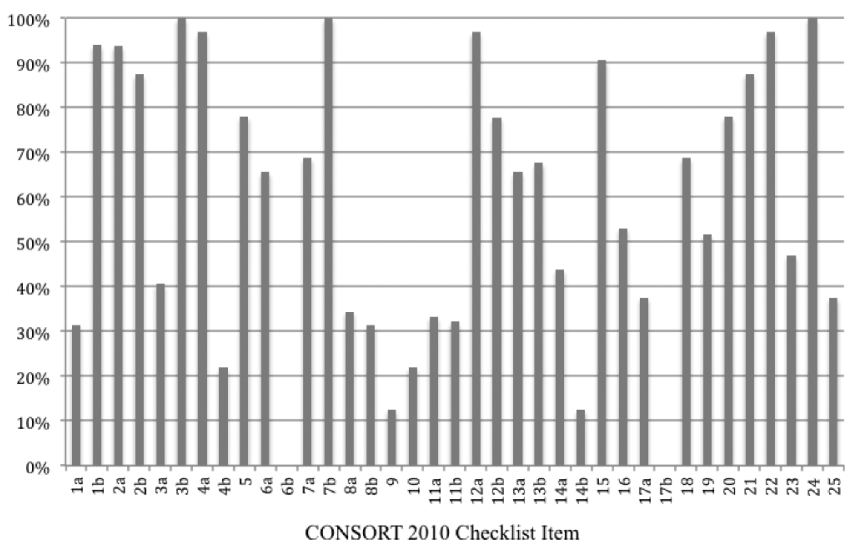

Abstract 3 Figure 1 Percentage of studies adequately reporting each CONSORT 2010 checklist item where applicable 